\title{
On the modeling of aiding mixed convection in vertical channels
}

\author{
Hua Sun · Ru Li · Eric Chénier · Guy \\ Lauriat
}

\begin{abstract}
This paper aims at showing that to prescribe a flow rate at the inlet section of a vertical channel with heated walls leads to surprising and counterintuitive physical solutions, especially when the problem is modeled as elliptical. Such an approach can give rise to the onset of recirculation cells in the entry region while the heat transfer is slightly increased under the influence of buoyancy force. We suggest an alternative model based on more realistic boundary conditions based on a prescribed total pressure at the inlet and a fixed pressure at the outlet sections. In this case, the pressure and buoyancy forces act effectively in the same direction and, the concept of buoyancy aiding convection makes sense. The numerical solutions emphasize the large differences between prescribed inlet velocity and solutions provided with our pressure based boundary conditions.
\end{abstract}

Keywords Aiding mixed convection - Vertical open channel flows - Computational fluid dynamics · Boundary conditions

\section{Nomenclature}

$\begin{array}{ll}a & \text { thermal diffusivity }\left[\mathrm{m}^{2} \mathrm{~s}^{-1}\right] \\ A & \text { aspect ratio, } A=H / D \\ c_{p} & \text { specific heat }\left[J \mathrm{~K}^{-1} \mathrm{~kg}^{-1}\right] \\ c_{x}, c_{z} & \text { stretching parameters, Eq. } 13 \text { and } 13 \\ D & \text { plate spacing }[\mathrm{m}] \\ D_{h} & \text { hydraulic diameter, } D_{h}=2 D[\mathrm{~m}] \\ g & \text { gravitational acceleration }\left[\mathrm{ms}^{-2}\right] \\ G r_{H} & \text { Grashof number based on } H, \\ & G r_{H}=g \beta_{0} \Delta T H^{3} / \nu_{0}^{2} \\ h & \text { heat transfer coefficient }\left[W \mathrm{~m}^{-2} K^{-1}\right] \\ H & \text { channel height }[\mathrm{m}]\end{array}$

Université Paris-Est, Laboratoire de Modélisation et Simulation Multi Echelle, MSME UMR 8208 CNRS, 5 bd Descartes, 77454 Marne-la-Vallée, France

E-mail: lauriat@univ-mlv.fr 


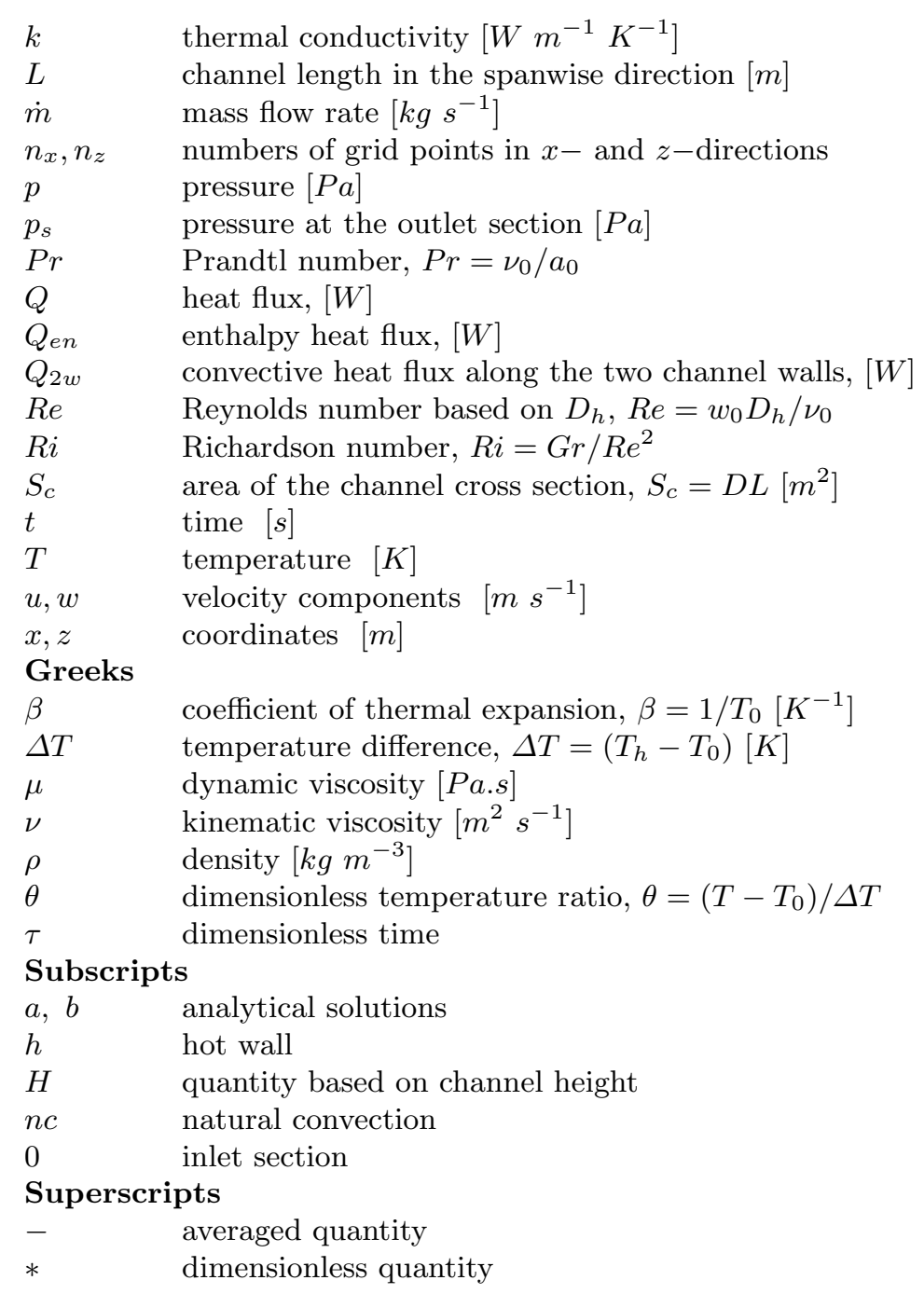

\section{Introduction}

Mixed convection may be defined as heat transfer situations where both pressure and buoyancy forces interact. In vertical channels, the bulk fluid flow can be either upward or downward, and the solutal and thermal buoyancy forces may be either assisting or opposing the forced flow. As a result, several hundred of papers were published just for mixed convection flows in vertical ducts of various shapes and thermal conditions, including two phase flows with surface condensation or evaporation, and flows in porous filled ducts. Most studies considered buoyancy assisted flow, i.e. upward flows with heating or downward flows with cooling. Under these conditions, the axial velocities may increase near the channel walls and decrease within the core region, with possible occurrence of a flow recirculation in the entry 
region for large effects of the buoyancy force [1-4] (not to be confused with flow reversal occurring close to the outlet section in asymmetrically heated channels $[5-10])$. In these cases, it is generally expected that heat transfer for mixed convection is larger than for forced convection. This intuition is obviously well physically based. However, numerical solutions may lead to opposite effects, not owing to the numerical accuracy but because the boundary conditions are not well specified. This paper is focused on this issue.

The extensive interest borne upon mixed convection in ducts stems from its wide range of practical applications including the design of compact heat exchangers, cooling of electronic equipment and, solar energy collectors. Numerous theoretical and experimental investigations were reviewed in many textbooks, such as in Aung [11], Gebhart et al. [12] or Bejan [13]. The present study is focused on mixed convection of air as the working fluid for typical geometries used for active cooling from the back of vertical photovoltaic cell panels operating at high fluxes.

This work considers the effect of thermal buoyancy force only (the effect of solutal force being similar, provided it acts in the same direction as the thermal force), and in the case of upward forced or natural flow direction (symmetrically heated vertical channel), commonly termed as buoyancy aiding or assisting mixed convective flows.

Most of the analytical and numerical studies on mixed convection were based on approximate problem formulations in order to easily solve the governing equations thanks to parabolized forms (or boundary-layer type equations for external flows with the streamwise pressure gradient dropped out [14]) which allow not to prescribe boundary conditions at the outlet duct section. On the other hand, the increasingly use of commercial computational fluid dynamics packages during the last decade has led to solve the problem when it is based on fully elliptic formulation, mostly by considering again prescribed inlet flow rate and outlet free boundary conditions. However, despite the large number of papers available in the current literature, the fundamental question of inlet/outlet flow conditions seems not to have been properly addressed because it appears that mixed convection with fixed inlet velocity (and thus Reynolds number) was very often considered as the relevant formulation for solving mixed convection.

The aim of this paper is at showing that the use of prescribed inlet velocity condition (constant flow rate whatever the importance of the buoyancy force) leads to completely different solutions in comparison with those obtained using prescribed total pressure inlet and pressure outlet conditions. For our viewpoint, such pressure boundary conditions appear to provide more realistic physical behaviors in practical applications, as it will be shown in what follows.

We are considering the practical case of air flowing in vertical flat-plate channels submitted to uniform wall temperatures, and maximum temperature differences compatible with the Boussinesq approximation. Although most of the results are presented in dimensional form, we also analyze the relevance of scaling predictions based on dimensionless parameters. It should be added that the extension of the present work to weakly compressible formulation (i.e. large temperature differences) is straightforward when using our in-house computational code, but the results obtained have shown that the main conclusions drawn in this paper remain unchanged. 


\section{Governing equations}

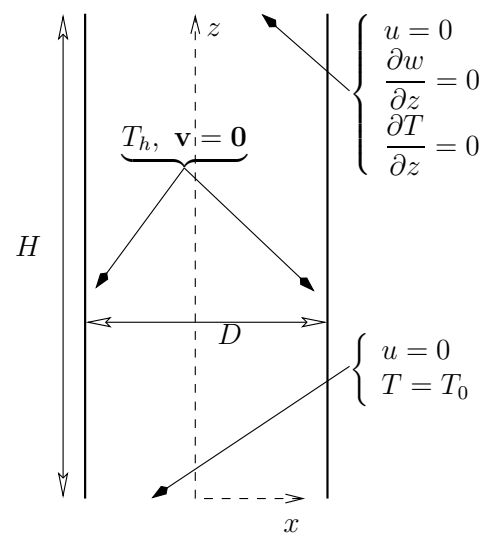

Fig. 1 The physical model of mixed convection in a vertical channel. The full boundary conditions are written in Eqs. (5-7) for the three cases investigated.

We consider two-dimensional, incompressible and laminar buoyancy-assisting flows inside a vertical parallel-plate channel, as shown in Fig. 1. The fluid enters the channel of height $H$ at ambient temperature and traverses upward, being heated by the hot walls at uniform temperature $T_{h}$. The fully developed region may be eventually reached at the outlet $(z=H)$, after a development length depending on the value of the plate spacing as well as on importances of the pressure and buoyancy forces. On account of the maximum temperature difference invoked, it is assumed that all physical properties are constant except for the density in the buoyancy force in the vertical direction (Boussinesq approximation). The reference temperature has been taken to be the inlet temperature, $T_{0}$, like in most of the solutions reported in the current literature. However, this choice remains an open question, as discussed by Barletta and Zanchini [15], especially for fully developed mixed-convection. Owing to the stability results by Chen and Chung [16], the governing equations are written in transient form in order to capture possible transitions to unsteady flows. With the $z$-axis pointing upwards and the origin of coordinates placed at the center of the inlet section, the conservation equations based on a fully elliptic model are

$$
\begin{gathered}
\nabla \cdot \mathbf{v}=0 \\
\rho_{0}\left(\frac{\partial \mathbf{v}}{\partial t}+\nabla \cdot(\mathbf{v} \otimes \mathbf{v})\right)=-\nabla\left(p+\rho_{0} g z\right)+\mu_{0} \nabla^{2} \mathbf{v}+\rho_{0} g \beta\left(T-T_{0}\right) \mathbf{e}_{z} \\
\frac{\partial T}{\partial t}+\nabla \cdot(\mathbf{v} T)=a_{0} \nabla^{2} T
\end{gathered}
$$

where $\rho_{0}, \mu_{0}$ and $a_{0}$ are the fluid density, dynamic viscosity and thermal diffusivity evaluated at $T_{0}$. 


\section{Boundary and initial conditions}

The initial condition is a fluid at rest at uniform temperature $T_{0}$.

The boundary conditions are written as follows:

- Along the vertical isothermal walls:

$$
\mathbf{v}=0, T=T_{h} \text { at } x= \pm D / 2 \text { and for } 0<z<H
$$

where $D$ and $H$ are the channel width and height, respectively.

- At the inlet $(z=0)$ and outlet $(z=H)$ sections for $-D / 2<x<D / 2$ :

(a) natural convection

$$
\begin{aligned}
& u=0, \frac{\partial w}{\partial z}=0, p=-\rho_{0} \frac{w^{2}}{2}, T=T_{0} \text { at } z=0 \\
& u=0, \frac{\partial w}{\partial z}=0, p+\rho_{0} g H=0, \frac{\partial T}{\partial z}=0 \text { at } z=H
\end{aligned}
$$

(b) forced or mixed convection

b1 : fixed flow rate ( $w_{0}$ prescribed $)$

$$
\begin{aligned}
& u=0, w=w_{0}, T=T_{0} \text { at } z=0 \\
& u=0, \frac{\partial w}{\partial z}=0, p+\rho_{0} g H=0, \frac{\partial T}{\partial z}=0 \text { at } z=H
\end{aligned}
$$

b2 : fixed inlet total pressure and outlet pressure $\left(p_{s}\right)$

$$
\begin{aligned}
& u=0, \frac{\partial w}{\partial z}=0, p=-\rho_{0} \frac{w^{2}}{2}, T=T_{0} \text { at } z=0 \\
& u=0, \frac{\partial w}{\partial z}=0, p+\rho_{0} g H=p_{s}, \frac{\partial T}{\partial z}=0 \text { at } z=H
\end{aligned}
$$

With boundary conditions Eq. 7 applied to forced or mixed convection, a pressure drop $\left(p_{s}<0\right)$ is prescribed at the outlet section (it could be as well an increase in pressure at the inlet section). Therefore, the boundary conditions are inlet temperature and outlet pressure, zero $z$-derivative for the vertical velocity component and zero horizontal velocity-component. It should be emphasized that these flow B.C. differ somehow from those used in most of previous works.

Let us emphasize that our results achieved using Eq. 6 or Eq. 7 are almost identical, provided the average velocities $\bar{w}(0)$ are the same. The choice of the particular boundary condition Eq. 6 may lead to wrong physical interpretations, because the imposed velocity $w_{0}$ is completely uncorrelated to the velocity induced by the natural convection flow. In contrast, the use of Eq. 7 preserves the coherence of the fluid flow with respect to free convection (Eq. 5) as it will be shown in what follows.

The governing equations are generally cast in dimensionless form by using either the set of dimensionless variables $X=x / D_{h}, Z=z / D_{h}, \theta=\left(T-T_{0}\right) /\left(T_{h}-\right.$ $\left.T_{0}\right), U=u / w_{0}, W=w / w_{0}$ and $P=p / \rho_{0} w_{0}^{2}$ or the set $X, Z=z /\left(D_{h} R e\right), \theta$, $U=D_{h} u /\left(\nu_{0}\right), W$ and $P$ where $D_{h}=2 D$ is the hydraulic diameter. It follows that the problem formulation depends on four parameters: $A=H / D, R e=w_{0} D_{h} / \nu_{0}$, $G r=g \beta\left(T_{h}-T_{0}\right) D_{h}^{3} / \nu_{0}^{2}=8 G r_{H} / A^{3}$ and $\operatorname{Pr}=\nu_{0} / a_{0}$, the channel aspect ratio, the Reynolds, Grashof and Prandtl numbers, respectively. The relative strength of the buoyancy force is then characterized either by the Richardson number, $R i=$ $G r / R e^{2}$, for the first set of dimensionless variables or by the product $R i \times R e=$ $G r / R e$ for the second set. Some controversy still exist about the best pertinent parameter, e.g. $R i$ or $R i \times R e$. 


\subsection{Heat transfer}

The total heat transfer rate released by two walls of depth $L$ and height $H$, denoted as $Q_{2 w}$, is calculated as follows:

$$
Q_{2 w}=\left.2 L k_{0} \int_{0}^{H} \frac{\partial T}{\partial x}\right|_{x=D / 2} d z=2 \bar{h}(L H)\left(T_{h}-T_{0}\right)
$$

where $L$ is the length of the channel in the y-direction, $\bar{h}$ is the mean heat transfer coefficient defined by the right equality in Eq. 8. For natural or mixed convection, a mean Nusselt number expressed by $\overline{N u}_{2 w}=Q_{2 w} /\left[2(L H)\left(k_{0} \Delta T / D\right)\right]$ may be introduced [17].

The enthalpy flux removed from the two heated surfaces by the stream is

$$
Q_{e n}=\rho_{0} c_{p} S_{c}\left[(\overline{w T})_{H}-(\overline{w T})_{0}\right]
$$

where $(\overline{w T})_{z}$ is the average of $(w T)$ over the channel cross-section of area $S_{c}=D L$. By using again $2(L H)\left(k_{0} \Delta T / D\right)$ as a reference heat flux, we can define a Nusselt number $\overline{N u}_{e n}=Q_{e n} /\left[2(L H)\left(k_{0} \Delta T / D\right)\right]$.

It should be emphasized that $Q_{2 w}$ is always greater than $Q_{e n}$ because the heat losses by axial diffusion through the channel inlet are not included into the definition of $Q_{e n}$. When the wall temperatures are kept fixed, $Q_{2 w}$ and $Q_{e n}$ merge provided that the Reynolds number is large enough in order that axial diffusion may be assumed negligibly small. The difference $Q_{\text {cond }}=Q_{2 w}-Q_{\text {en }}$ yields the effect of axial diffusion which can be written as

$$
\begin{aligned}
Q_{\text {cond }} & =\left.k_{0} \int_{-D / 2}^{D / 2} \frac{\partial T}{\partial z}\right|_{z=0} d x \\
& =\left.2 k_{0} \int_{0}^{H} \frac{\partial T}{\partial x}\right|_{x=D / 2} d z-\rho_{0} c_{p} \int_{-D / 2}^{D / 2}\left[w(x, H) T(x, H)-w(x, 0) T_{0}\right] d x
\end{aligned}
$$

For negligible axial heat diffusion, equation (8) reads:

$$
\left.2 k_{0} L \int_{0}^{H} \frac{\partial T}{\partial x}\right|_{x=D / 2} d z=2 \bar{h}(H L)\left(T_{h}-T_{0}\right)=\dot{m} c_{p}\left(T_{b}(H)-T_{0}\right)
$$

where $\dot{m}=\rho_{0} \bar{w}_{0}(D L)$ and $T_{b}(H)=(\overline{w T})_{H} / \bar{w}_{0}$ is the bulk temperature at the outlet section. Such an approximation is one of the foundations introduced for establishing the analytical solutions reported in Ref. [13, 17, 18].

\section{Numerical method and validation}

The mass, momentum and energy conservation equations were spatially discretized by using the collocated finite volume method presented in [19, 20], applied here for structured meshes. The key features of this method are:

- the normal fluxes to cell faces are defined by a consistent two-points approximation, 
- the variables transported by the fluid flow are expressed on cell faces in a centered manner, not by using a linear interpolation at the faces,

- the "pressure gradient" is defined by the dual expression of the velocity divergence and therefore, it can be non-consistent with a gradient.

An implicit second-order Euler scheme was adopted for time derivatives at time $t=(n+1) \Delta t$, with an implicit treatment of the diffusion terms and an AdamsBashforth extrapolation procedure for the transport terms. A stabilization method is necessary to prevent from the onset of checkerboard oscillations associated with collocated schemes. This is performed through the velocity-pressure decoupling which is handled by a projection method [21].

Attention was also paid to the temperature discontinuities at the corner of the inlet section (i.e. at $x= \pm D / 2, z=0$ ). In the case of pure conduction, the analytical heat fluxes along the vertical walls are indeed infinite. Therefore, the wall heat fluxes calculated numerically, $Q_{2 w}$, cannot converge when refining the meshes (see [22] for details). To avoid this problem, it is then necessary to smooth the thermal boundary conditions in order to recover some regularity. To this end, we have substituted the uniform temperature imposed at the walls by a temperature distribution which decreases abruptly towards $T_{0}$ close to $z=0$. The steep function retained in this work writes:

$$
T_{p}(z)=T_{0}+\left(T_{h}-T_{0}\right) \tanh \left(\Gamma \frac{z}{H}\right)
$$

For example, by choosing $\Gamma=1000$, the wall temperature $T_{p}(z)$ for a channel height $H=1 \mathrm{~m}$ varies from $0.5 T_{h}$ at $z \simeq 0.55 \mathrm{~mm}$ to $0.9 T_{h}$ at $z \simeq 1.5 \mathrm{~mm}$ and then becomes larger than $0.99 T_{h}$ for $z>2.65 \mathrm{~mm}$. We checked that such a steep variation in $T_{p}(z)$ does change significantly the temperature field while it allow second order convergence of the wall heat fluxes, $Q_{2 w}$.

The velocity components, the temperature field and the pressure correction were calculated by using the Bi-Conjugate Gradient Stabilized (BCGS) method, preconditioned by an incomplete LU decomposition. Owing to the axial symmetry of the flow, the problem was solved on the half-width of the channel. The faces of the mesh $x_{i}^{f}$ and $z_{k}^{f}$ are defined as follows:

$$
\begin{aligned}
& \frac{x_{i}^{f}}{D / 2}=\frac{i}{n_{x}}-\frac{c_{x}}{2 \pi} \sin \left(\frac{i}{n_{x}} \pi\right), \quad 0 \leq i \leq n_{x} \\
& \frac{z_{k}^{f}}{H}=\frac{\tanh \left(c_{z}\left(\frac{k}{n_{z}}-1\right)\right)}{\tanh \left(c_{z}\right)}+1, \quad 0 \leq k \leq n_{z}
\end{aligned}
$$

The coefficients $c_{x}$ and $c_{z}$ define the mesh refinements in the entrance region and along the isothermal wall. By choosing $c_{x}=1$ and $c_{z}=1.82$, the size ratios between the largest cell and the smallest cell are equal to 3 and 10 in horizontal and vertical directions, respectively. The time integration was performed with the Courant-Friedrich-Levy number kept fixed to $C F L=0.5$.

The asymptotic behavior of the numerical solution was investigated using a Richardson extrapolation on one set of the three meshes defined by $n_{x} \in$ $\{10,20,40\}$ with $n_{z}=20 n_{x}$. This method leads to the extrapolated value of $f$ which is written

$$
f_{\text {Extrap. }}=f_{n_{x}}+\frac{c_{n_{x}}}{\left(n_{x}\right)^{s}}
$$


where $c_{n_{x}}$ is a coefficient depending on $n_{x}$ and $f_{n_{x}}$ is the numerical result on grid $n_{x} \times n_{z}$. For sufficiently fine meshes, the value of $s$ must tend towards the order of consistency of the numerical scheme (i.e. $s=2), f_{\text {Extrap. }}$ must then become independent of the mesh. For natural convection flow, the mesh $20 \times 400$ defined for a half width of the channel provides accurate results within 3 digits (Tab. 1). This grid size is then retained for all computations.

\begin{tabular}{c|ccc||c|c}
$n_{x}$ & 10 & 20 & 40 & $s$ & $f_{\text {Extrap. }}$ \\
\hline$Q_{2 w}(W)$ & 130.39 & 130.31 & 130.30 & 2.66 & 130.30 \\
$Q_{e n}(W)$ & 130.32 & 130.22 & 130.20 & 2.01 & 130.19 \\
$\bar{w}_{n c}(m / s)$ & 0.3840 & 0.3833 & 0.3831 & 2.01 & 0.3830
\end{tabular}

Table 1 Convective heat flux, enthalpy heat flux and mean velocity according to the mesh refinement $\left(n_{z}=20 n_{x}\right)$, for $D / 2=1 \mathrm{~cm}$ and $H=1 \mathrm{~m}$. Order $s$ of the numerical scheme and Richardson-extrapolated values (see Eq. 14).

\section{Results}

The influence of the dynamical boundary conditions at the inlet and outlet sections is discussed by considering air entering at $T_{0}=300 \mathrm{~K}$ into a vertical channel with height walls $H=1 \mathrm{~m}$ at uniform hot temperature $T_{h}=320 \mathrm{~K}$ (or $H=1.5 \mathrm{~m}$, just for allowing comparisons with previously published works $[2,3]$ ). The channel width was $D=2 \mathrm{~cm}$ or $D=3 \mathrm{~cm}$. With the air properties taken at $T_{0}=$ $300 K\left(\rho_{0}=1.176 \mathrm{~kg} \cdot \mathrm{m}^{-3}, \mu_{0}=1.8510^{-5} \mathrm{~Pa} . \mathrm{s}, c_{p, 0}=1006 \mathrm{~J} . \mathrm{kg}^{-1} \cdot \mathrm{K}^{-1}, k_{0}=\right.$ $\left.0.0261 \mathrm{~W} \cdot \mathrm{m}^{-1} \cdot \mathrm{K}^{-1}\right)$ the Grashof number is then $\mathrm{Gr}_{H}=2.6410^{9} \mathrm{H}^{3}$. Channel heights less than about two meters are thus considered as maximum in order to keep a laminar flow.

For pure natural convection (Tab. 1), the computations yield an average velocity $\bar{w}_{c n}=0.38 \mathrm{~m} / \mathrm{s}$ and a pressure drop at the inlet section (Eq. 5) $\bar{p}_{c n, i n}=$ $-0.087 \mathrm{~Pa}$. The heat flux transferred along one of the isothermal walls is $Q_{1 w}=$ $Q_{2 w} / 2=65.2 \mathrm{~W}$ by unit of depth length (direction perpendicular to the plane of Fig. 1). These values are the basis for the discussions of mixed convection results presented in what follows.

Before getting further into the result section, let us recall that flow solutions are similar provided that the average velocities, imposed or resulting from computation, are identical. However, when a constant flow rate is prescribed, counterintuitive results or surprising phenomena may arise. Such situations, developed in details in Sec.4.1, are now briefly exemplified. By using Eq. 6, astonishing flow recirculations are highlighted close to inlet section. Their occurrences are simply due to the incompatibility between the imposed flow rate and the necessity of feeding the dynamic boundary layers produced by the buoyancy along the vertical heated walls. Similarly from a purely thermal viewpoint, it is also expected that the buoyant force increases the velocities in the boundary layers and then improves the heat transfer at the solid walls. However this behavior is not observed when the inlet velocity is prescribed. 
The next two subsections are then devoted to the detailed analysis of the fluid flows and heat transfers when using boundary conditions 6 and 7. For both sets of boundary conditions, comparisons between natural, forced and mixed convection flows are performed. These comparisons emphasize clearly that only the pressure boundary conditions make sense.

\subsection{Constant flow rate}

We are first considering prescribed inlet velocity in the range $0.1 \mathrm{~m} / \mathrm{s} \leq w_{0} \leq$ $1 \mathrm{~m} / \mathrm{s}$ in order to satisfy the assumption of laminar flow $\left(R e=2500\right.$ for $w_{0}=$ $1 \mathrm{~m} / \mathrm{s}$ ). For pure forced convection, it results that the difference in pressure between the inlet and outlet sections is within $\Delta p=0.061 \mathrm{~Pa}$ and $\Delta p=0.96 \mathrm{~Pa}$.

The $\Delta p$-variations versus the inlet velocity are shown in Fig. 2 for forced convection and for mixed convection. Since the outlet pressure has been fixed to $p_{s}=0$ (see Eq. 6), that implies $\Delta p=\bar{p}(0)$. For forced convection, the inlet pressure is always positive while it is negative for mixed convection if the inlet velocity is smaller than the inlet velocity corresponding to pure natural convection $\left(\bar{w}_{n c}=0.38 \mathrm{~m} / \mathrm{s}\right)$. Therefore, the pressure and buoyancy forces are opposite if $w_{0}<\bar{w}_{n c}$.

Let us now consider computations based on the dimensionless form of the governing equations. In most of the papers dealing with aiding mixed convection, the Reynolds and Grashof numbers were arbitrarily fixed: the relative importance of one convective mode was discussed by considering only the value of $R i$ or $R i \times R e$ with the same length scale for $R e$ and $G r$. For the present computations $(D=$ $2 \mathrm{~cm})$, it is found that $R i=2.6210^{-2} / w_{0}^{2}\left(w_{0}\right.$ in $\left.\mathrm{m} / \mathrm{s}\right)$ and $R i \times R e=66.4 / w_{0}$. Therefore, either the first or the second criterion (e.g $R i>\mathcal{O}(1)$ [13] or $R i \times$ $\left.R e>\mathcal{O}\left(10^{3}\right)[23]\right)$ leads to assume that natural convection dominates for $w_{0}$ less than about $0.15 \mathrm{~m} / \mathrm{s}$, a value smaller than $\bar{w}_{c n}=0.38 \mathrm{~m} / \mathrm{s}$. For the practical case considered, the average velocity $0.15 \mathrm{~m} / \mathrm{s}$ can only be reached for a negative pressure gradient (Fig. 2), namely for a "forced convection" opposing to the natural convection. Thus, concluding that natural convection is dominating has no physical meaning since the pressure gradient plays a dominant part into the flow rate.

The streamlines plotted in Fig. 3 shows another view of the results linked to a fixed flow rate smaller than the natural inlet flow rate. For this case, we consider a channel height $H=1.5 \mathrm{~m}$ and a channel width $D=3 \mathrm{~cm}$ in order to check that the present results are in full agreement with those published in [2-4].

Figure 3 raises obviously the question of the origin of existence of flow recirculations within the entry region for aiding mixed convection in vertical channels. As it is well established nowadays, the separation bubble (the axis of which being the channel axis) cannot be predicted when using parabolized formulations. On the other hand, elliptical models may lead to such flow topologies provided that the inlet velocity is prescribed without any reference to the natural convection velocity. The case shown in Fig. 3 corresponds to a clear recirculating flow, easily reproducible by using the simulation conditions reported in the legend of this figure. The conclusion is that the flow field referenced as "buoyancy-assisted mixed flow" is similar to the flow predicted for "opposing mixed convection" with $p_{s}=1.4 \mathrm{~Pa}(\Delta p<0)$.

Figure 4 shows the variations of the vertical velocity component along the centerline (e.g. $w(0, z))$ for the three convective modes. For forced convection, $w(0, z)$ 


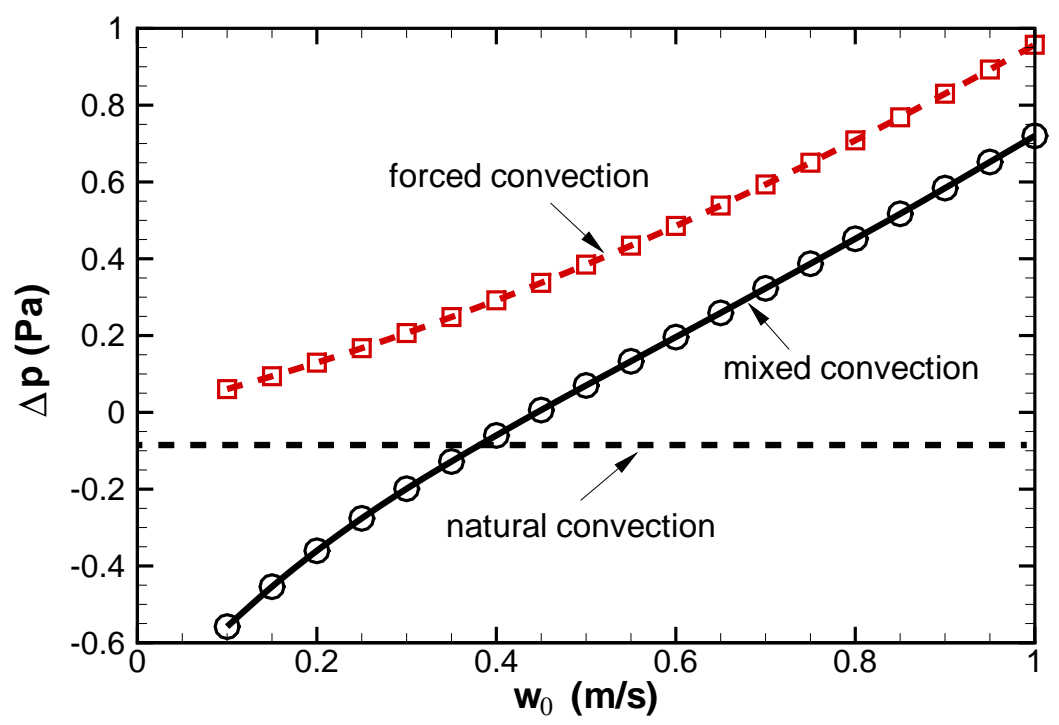

Fig. 2 Variations of the pressure difference between inlet and outlet sections according to the prescribed inlet velocity for forced, mixed and natural convection.

is the maximum flow velocity at any $z$-section and it increases upstream until the flow regime is fully established (before $z=H$ if $w_{0}<0.2 \mathrm{~m} / \mathrm{s}$ in the case considered). For mixed convection, the buoyancy force produces maximum in velocities near the walls. Therefore, $w(0, z)$ for mixed convection is lower than for forced convection when the flow rate is prescribed, the difference increasing upstream with $w_{0}$. If $w_{0}<\bar{w}_{n c}\left(\bar{w}_{n c}\right.$ being the average velocity for natural convection) the decrease in $w(0, z)$ at the bottom of the channel indicates a possible onset of flow recirculation (see Fig. 3). For $w_{0}=0.1 \mathrm{~m} / \mathrm{s}$ and $0.2 \mathrm{~m} / \mathrm{s}$, a stagnant zone characterized by a decrease in the axial velocity spreads within the lower half-region. This stagnation zone becomes more and more confined close to the channel inlet as the difference between $w_{0}$ and the natural convection velocity increases.

Figure 5 displays the variation in the total heat flux transferred at one of the channel walls $\left(Q_{1 w}=Q_{2 w} / 2\right)$ as function of the inlet velocity, the horizontal dashed line being for pure natural convection. As can be seen, mixed convection with a prescribed inlet velocity lower than that for pure natural convection leads to a lower heat transfer rate. In addition, the very small differences between the results for forced and mixed convection clearly show that the buoyancy force does not assist significantly the pressure force. For the lowest inlet velocity considered here $\left(w_{0}=0.1 \mathrm{~m} / \mathrm{s}\right)$, the Péclet number is $P e \approx 259$. Therefore, axial conduction is negligibly small $\left(Q_{\text {cond }} \ll Q_{2 w}\right.$, Eq. 8 and 10$)$. 


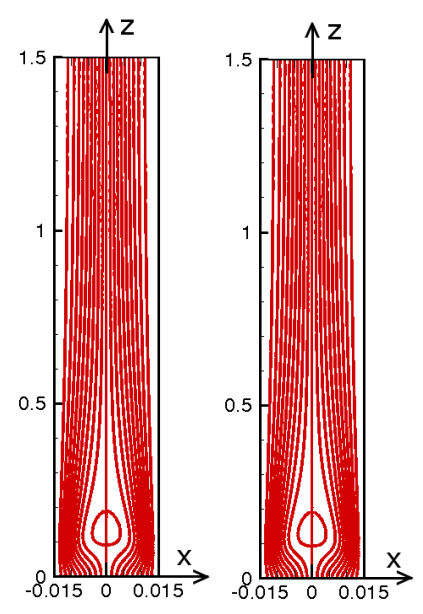

(a)

(b)

Fig. 3 Flow recirculation for mixed convection (a) prescribed inlet velocity, $w_{0}=0.05 \mathrm{~m} / \mathrm{s}$ (b) overpressure at the outlet section $p_{s}=1.4 \mathrm{~Pa}(\mathrm{D}=3 \mathrm{~cm}, H=1.5 \mathrm{~m}, \Delta T=20 \mathrm{~K})$.

\subsection{Pressure driven mixed convection}

When a pressure drop is prescribed at the outlet section $\left(p_{s}<0\right)$, with an inlet total pressure fixed, the pressure and the velocity profile at the inlet as well as the flow rate are not a priori known. Therefore, it is not possible to calculate the Reynolds and Richardson numbers until the end of the computations. However, the flow rate is necessary greater than that for pure natural convection. For the practical case investigated $(H=1 \mathrm{~m}, D=2 \mathrm{~cm}, \Delta T=20 \mathrm{~K})$, the maximum pressure (at the center of the inlet section) for natural convection is $p(0,0)=$ $-0.143 P a$. The inlet pressure for mixed convection should be lower than this value since the flow rate is assumed to increase $\left(\bar{p}(0) \approx-\rho_{0} \bar{w}^{2} / 2\right)$.

Figure 6 shows the axial variation of pressure along the channel axis. For natural convection the pressure minimum is at $z=0.24 \mathrm{~m}$ while it is located at the outlet section for forced convection. For mixed convection, the pressure curves exhibit a minimum, whose location is indicated by an arrow in Fig. 6. Therefore, $\partial p / \partial z$ is zero at this $z$-coordinate where the buoyancy force compensates viscosity and advection. When the pressure drop at the outlet section exceeds $0.6 \mathrm{~Pa}$, the pressure decreases monotonously from the inlet to the outlet section, indicating the predominance of forced convection.

The average velocity is displayed in Fig 7 versus the outlet pressure drop. In contrast to what has been found for prescribed inlet velocity, $\bar{w}$ for mixed convection is systematically greater than for forced convection: buoyancy force assists thus the pressure force whatever $p_{s}<0$ and, we can effectively speak 


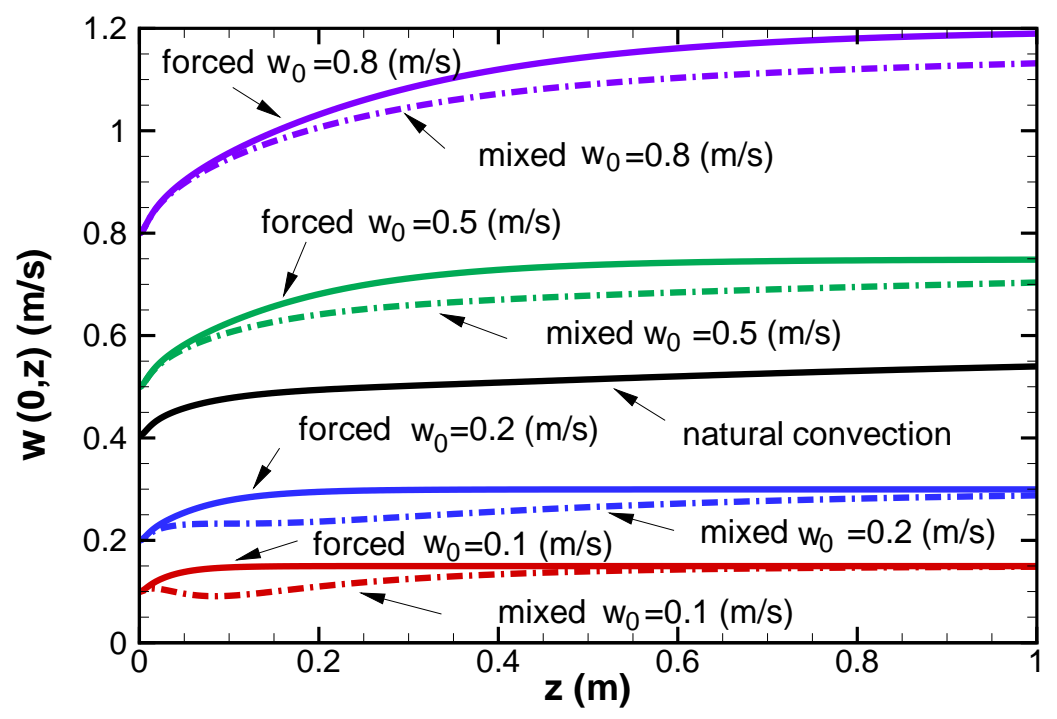

Fig. 4 Vertical velocity variations along the channel axis for forced and mixed convection according to the prescribed inlet velocity. The natural convection curve is also shown $(D=$ $2 \mathrm{~cm}, H=1 \mathrm{~m}, \Delta T=20 \mathrm{~K})$.

in terms of "buoyancy assisted mixed convection". The results is obviously an increase in the heat flux transferred along the walls as can be seen in Fig 8. The computations were not pursued for $p_{s}<-1.5 \mathrm{~Pa}$ owing to the large values of the Reynolds number involved $\left(R e=2744\right.$ for $p_{s}=-1.5 \mathrm{~Pa}$ ). Figure 8 shows that natural convection dominates for the smallest values of $-p_{s}$ and that forced convection dominates for the largest values of $-p_{s}$. The comparison between Fig. 5 (B.C. 6) and Fig. 8 (B.C. 7) clearly exhibits the large differences in the wall heat flux according to the type of boundary conditions. It should be noted that the forced convection curve does not tend to zero as $p_{s} \rightarrow 0$ because the problem turns then into a pure conduction problem. From Eq. 10 it is found that $Q_{\text {cond }}=4.8 \mathrm{~W}$ for $p_{s}=0$.

Let us now discuss the above results in terms of $R i$ or $R i \times R e$ ( $R e$ and $G r$ being both based on the same length scale, i.e $D_{h}$ ). When increasing the pressure drop, $R i$ and $R i \times R e$ decrease since the flow rate is increased. From $p_{s}=-0.1 P a$ to $p_{s}=-1.5 P a, R i$ decreases from 0.134 to 0.022 while $R i \times R e$ decreases from about 151 to about 57 . The criterion for the transition from dominant forced convection to dominant natural convection being $R i>\mathcal{O}(1)$ [13] or $R i \times R e>\mathcal{O}\left(10^{3}\right)$ [23], it could be assumed that natural convection plays a negligible role for the results discussed herein. In fact, the heat flux at one of the vertical walls is $Q_{1 w} \approx 65 \mathrm{~W}$ for pure natural convection. That substantially differs from $Q_{1 w} \approx 75 \mathrm{~W}$ for mixed convection when $p_{s}=-0.2 \mathrm{~Pa}\left(Q_{1 w} \approx 50 \mathrm{~W}\right.$ for forced convection). Therefore, any of these two criteria based on approximate analyses appears to be relevant. 


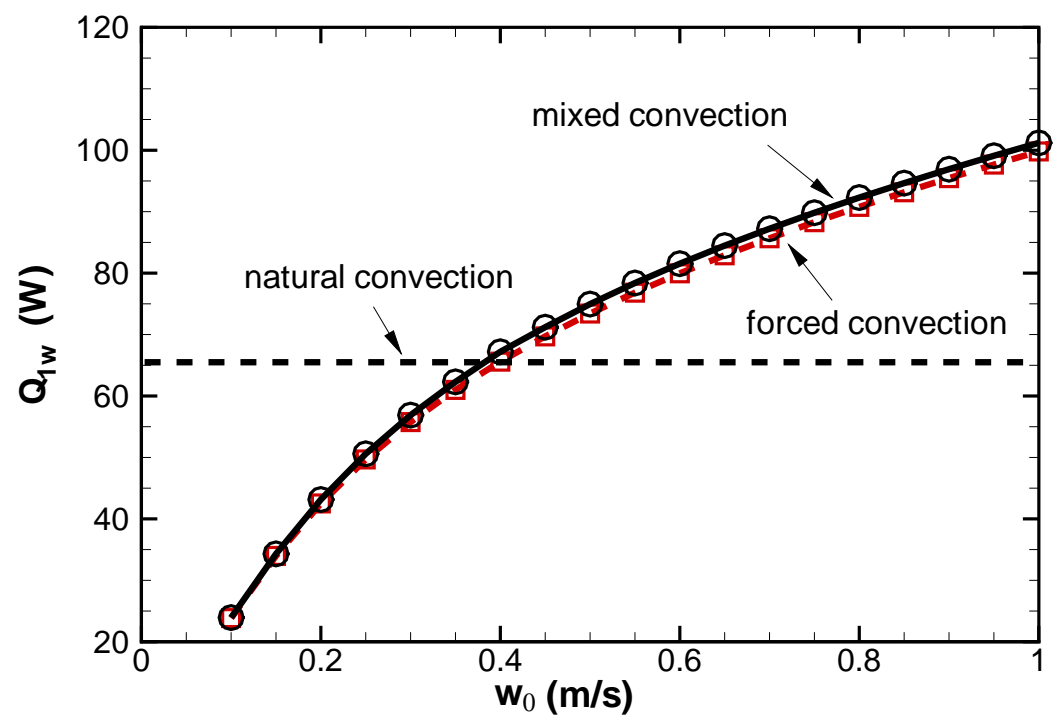

Fig. 5 Total heat flux transferred at one of the hot walls for forced and mixed convection according to the prescribed inlet velocity. The value for natural convection is also shown $(D=2 \mathrm{~cm}, H=1 \mathrm{~m}, \Delta T=20 \mathrm{~K})$.

The temperature distribution along the channel axis is plotted in Fig. 9 according to the pressure at the channel outlet section. Small increases in the axial temperature indicate that the channel flow regime turns into a boundary layer type regime. Asymptotic solutions for assisting mixed convection along a vertical isothermal plate may be thus useful. The present results show that it is not the case, at least for laminar flows (i.e. $\left|p_{s}\right|<1.5 \mathrm{~Pa}$ ). In addition, temperature as well as velocity profiles do not exhibit boundary layer-type behavior. When $p_{s}$ is decreased from $p_{s}=0$ (pure natural convection) to $p_{s}=-1.5 \mathrm{~Pa}$ (dominant forced convection), Figure 9 indicates a significant decrease in the axial temperature which is closely linked to an increase in heat transfer at the walls. Since the temperature increases up to the outlet section, an established velocity field cannot be predicted, as shown in Fig. 10. This figure exhibits also that the dynamical entry length augments with the Reynolds number. However, the usual criterion for defining the dynamical and thermal entry lengths for forced convection in a flat plate channel $\left(z_{d} \approx 410^{-4} R e_{D_{h}}\right.$ and $z_{t} / D_{h} \approx 0.01 R e_{D_{h}} \operatorname{Pr}$, respectively) are not met for the largest pressure drop considered.

\section{Conclusion}

Mixed-convection for assisting flows occurring in isothermal flat-plate channels was studied by solving numerically the system of conservation equations written in elliptical form. The computations were carried out by using a finite-volume 


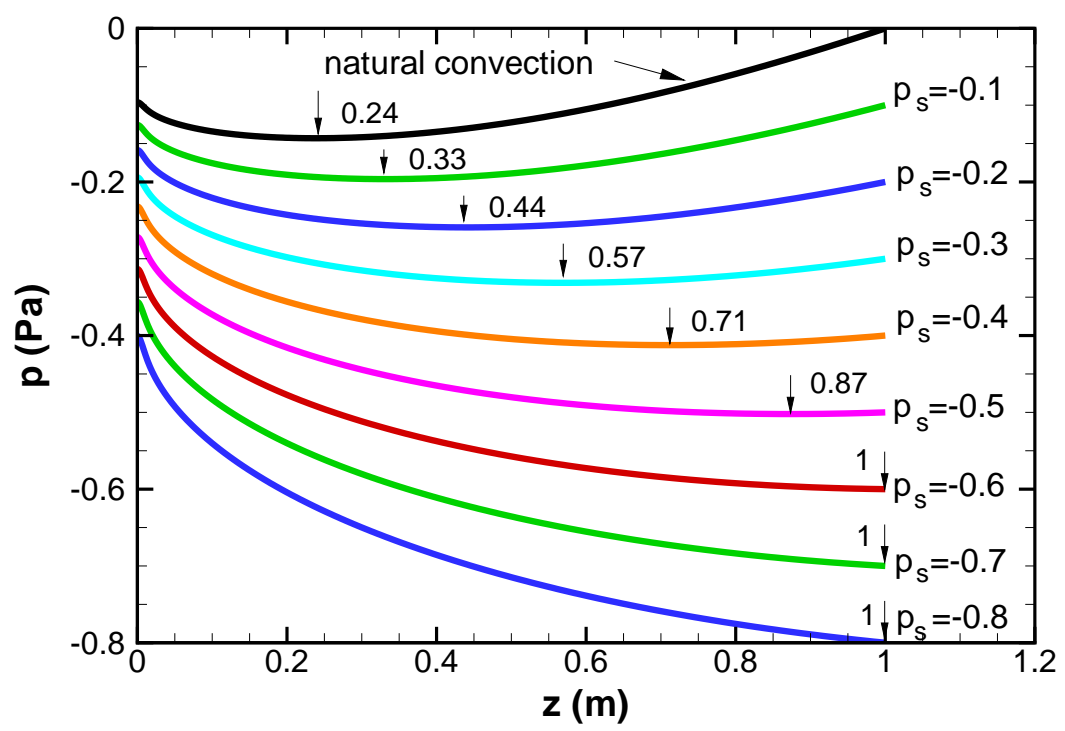

Fig. 6 Axial variation of pressure along the channel axis according to the outlet pressure drop $(D=2 \mathrm{~cm}, H=1 \mathrm{~m}, \Delta T=20 \mathrm{~K})$. The arrows indicate the positions (in $\mathrm{m}$ ) of the pressure minimum.

method implemented in an in-house code. The spatial order of convergence of the method and the accuracy of the results have been checked thanks to the Richardson extrapolation method and regularized inlet thermal conditions in order to properly solve cases of low flow rates linked to small plate spacings and/or small temperature differences.

As an example, we have considered air at ambient temperature flowing in vertical channels (height $H=1 \mathrm{~m}$ or $H=1.5 \mathrm{~m}$, width $D=2 \mathrm{~cm}$ or $D=$ $3 \mathrm{~cm}$ ). For a maximum temperature difference compatible with the Boussinesq approximation $(\Delta T=20 \mathrm{~K})$, leading to $G r_{H}=2.6510^{9} \mathrm{H}^{3}$, the entire laminar mixed-convection regime has been covered by changing either the inlet velocity or the outlet pressure drop. $R e_{D_{h}}$ was varied from $R e_{D_{h}} \approx 200$ to $R e_{D_{h}} \approx 2800$. Although most of the results have been discussed in their dimensional forms, we have also analyzed the reliability of scaling predictions based on dimensionless numbers.

The results for a prescribed inlet velocity $\overline{w_{0}}$ show that flow recirculations may appear in the entry region when the inlet velocity is lower than that for pure natural convection. In that case, the pressure force opposes to the buoyancy force and the problem is, in fact, similar to that of opposing natural convection with a downward bulk flow. In addition, we have shown that to prescribe $\overline{w_{0}}$ yields no significant difference between the wall heat fluxes calculated either for forced convection or for mixed convection.

The modeling of mixed convection based on a pressure drop at the outlet section $\left(p_{s}<0\right)$ and a total pressure at the inlet section leads to a completely 


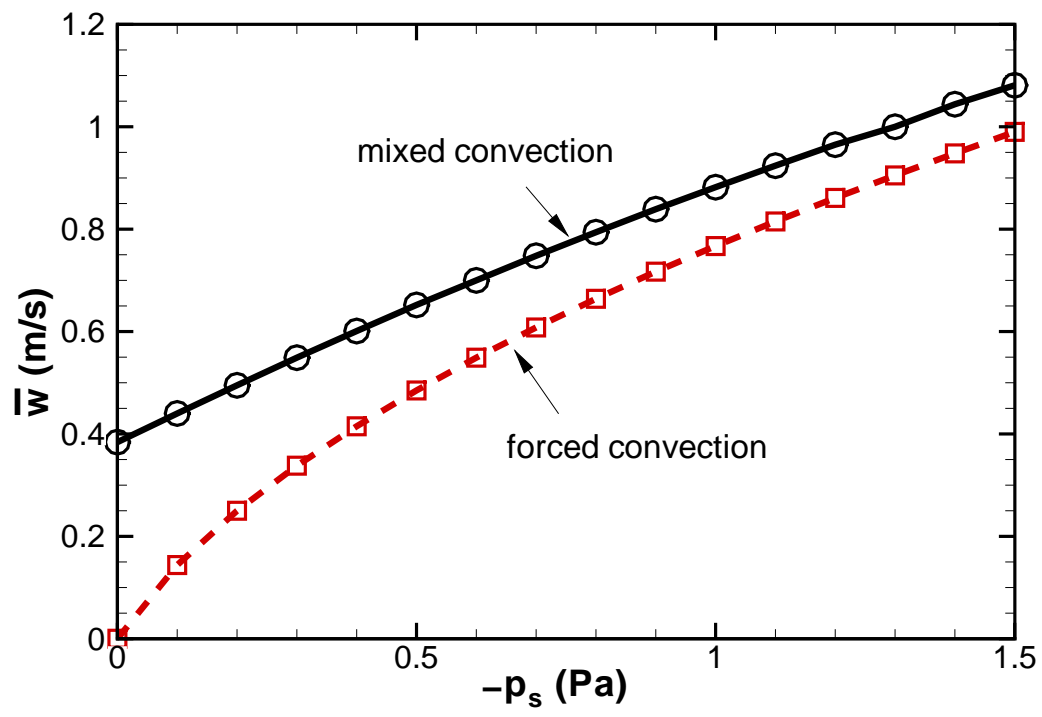

Fig. 7 Variations of the average velocity according to the pressure drop at the outlet section for forced and mixed convection $(D=2 \mathrm{~cm}, H=1 \mathrm{~m}, \Delta T=20 \mathrm{~K})$.

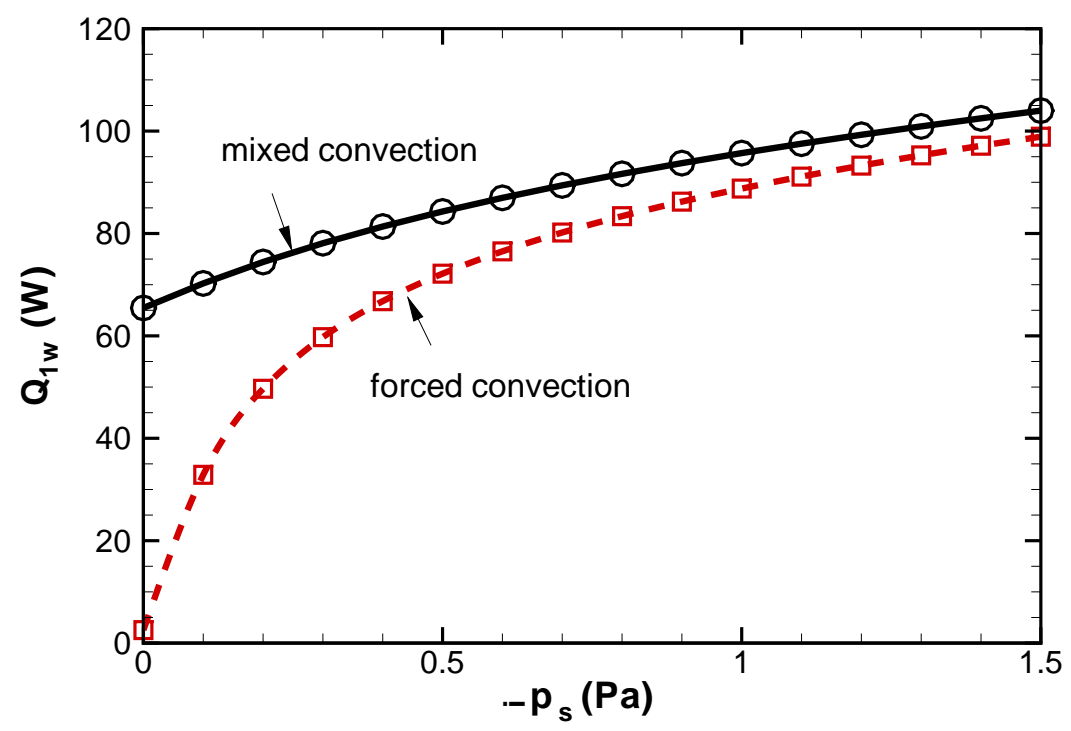

Fig. 8 Variations of the heat flux transferred at one of the walls according to the pressure drop at the outlet section for forced and mixed convection $(D=2 \mathrm{~cm}, H=1 \mathrm{~m}, \Delta T=20 \mathrm{~K})$. 


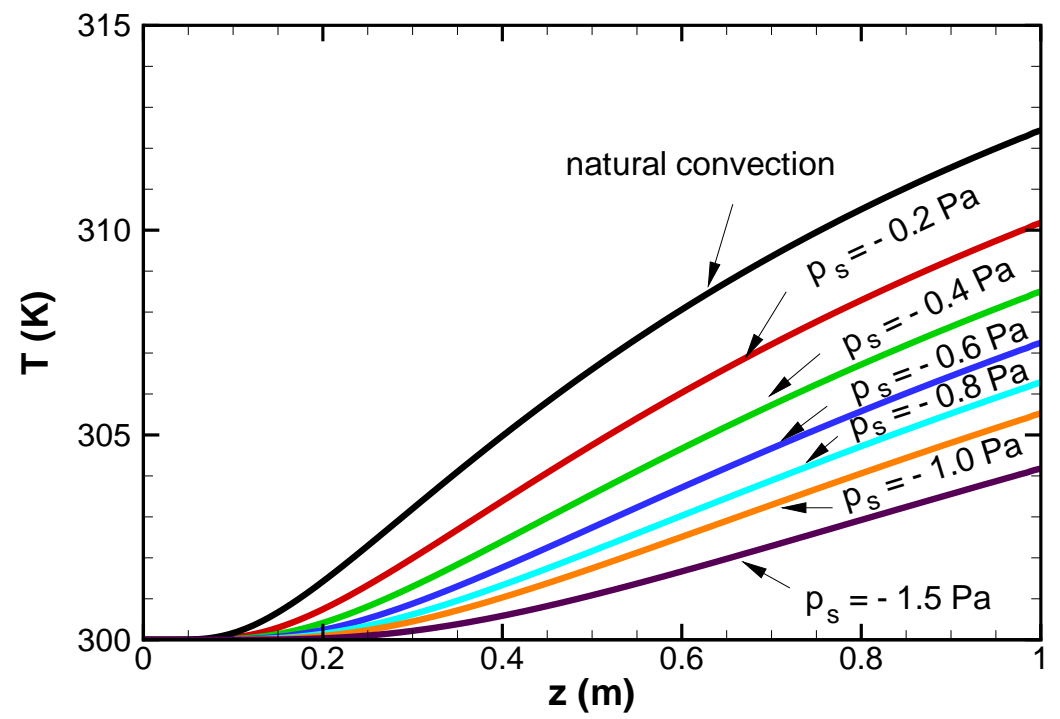

Fig. 9 Variations of the temperature along the channel axis according to the pressure drop at the outlet section for natural and mixed convection $(D=2 \mathrm{~cm}, H=1 \mathrm{~m}, \Delta T=20 \mathrm{~K})$.

different analysis of the results, with a better accordance with the common physical sense. When imposing an outlet pressure drop, natural convection assists effectively forced convection and the flow rate as well as the wall heat fluxes are systematically increased over those found for pure forced convection.

\section{References}

1. D. B. Ingham, D. J. Keen, P. J. Heggs, B. R. Morton, Recirculating pipe flows, J. Fluid Mech. 213 (1990) 443-464.

2. Z. Ait Hammou, B. Benhamou, N. Galanis, J. Orfi, Laminar mixed convection of humid air in a vertical channel with evaporation or condensation, Int. J. Therm. Sci. 43 (2004) 531-539.

3. G. Desrayaud, G. Lauriat, Flow reversal of laminar mixed convection in the entry region of symmetrically heated, vertical plate channels, Int. J. Therm. Sci. 48 (2009) 2036-2045.

4. N. Laaroussi, G. Lauriat, G. Desrayaud, Effects of variable density for film evaporation on laminar mixed convection in a vertical channel, Int. J. Heat Mass Transfer 52 (2009) 151-164.

5. W. Aung, G. Worku, Theory of fully developed, combined convection including flow reversal, ASME J. Heat Transfer 108 (1986) 485-488.

6. W. Aung, G. Worku, Developing flow and flow reversal in a vertical channel with asymmetric wall temperatures, ASME J. Heat Transfer 108 (1986) 289304. 


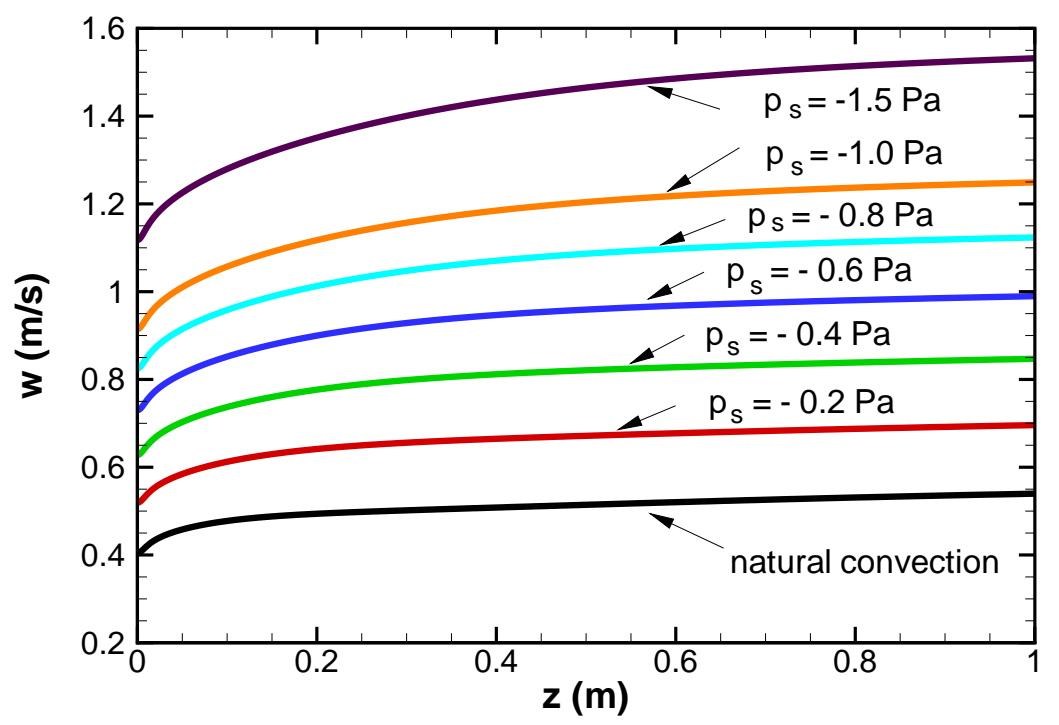

Fig. 10 Variations of the vertical velocity component along the channel axis according to the pressure drop at the outlet section for natural and mixed convection $(D=2 \mathrm{~cm}, H=1 \mathrm{~m}$, $\Delta T=20 \mathrm{~K})$.

7. C. Gau, K. Yih, W. Aung, Measurements of heat transfer and flow structure in heated vertical channels with buoyancy assisted and opposed flows, AIAA J. Thermophys. Heat Transfer 6 (1992) 707-712.

8. C. Gau, K. Yih, W. Aung, Reversed flow structure and heat transfer measurements for buoyancy-assisted convection in a heated vertical duct, ASME J. Heat Transfer 114 (1992) 928-935.

9. C. H. Cheng, S. Huang, W. Aung, Enhancement of flare method for predicting buoyancy-induced flow reversal in vertical ducts via parabolic model, Numer. Heat Transfer, Part B 31 (1997) 327-345.

10. C. S. Yang, D. Z. Jeng, K. A. Yih, C. Gau, W. Aung, Numerical and analytical study of reversed flow and heat transfer in a heated vertical duct, J. Heat Transfer 131 (2009) 072501.

11. W. Aung, Mixed convection in internal flow, Handbook of Single-Phase Convective Heat Transfer,S. Kakaç, R. K. Shah, W. Aung, eds, John Wiley and Sons, New York (1987)

12. B. Gebhart, Y. Jaluria, R. L. Mahajan, B. Sammakia, Buoyancy Induced Flows and Transport, Springer-Verlag (1988)

13. A. Bejan, Convection Heat Transfer, 3rd ed., Wiley, Hoboken (2004).

14. T. S. Chen, B. F. Armaly, W. Aung, Natural Convection: Fundamentals and Applications, Washington: Hemisphere Pub. Corp., eds: S. Kakaç, W. Aung and R. Viskanta (1985) pp. 699-725.

15. A. Barletta, E. Zanchini, On the choice of the reference temperature for fullydeveloped mixed convection in a vertical channel, Int. J. Heat Mass Transfer 
42 (1999) 3169-3181.

16. Y. C. Chen, J. N. Chung, The linear stability of mixed convection in a vertical channel flow, J. Fluid Mech. 325 (1996) 29-51.

17. A. Bar-Cohen, W. M. Rohsenow, Thermally optimum spacing of vertical, natural convection cooled, parallel plates, ASME J. Heat Transfer 106 (1984) $116-123$.

18. A. Bejan, E. Sciubba, The optimal spacing of parallel plates cooled by forced convection, Int. J. Heat Mass Transfer 35 (1992) 3259-3264.

19. E. Chénier, R. Eymard, O. Touazi, Numerical results using a colocated finite volume scheme on unstructured grids for incompressible fluid flows, Numer. Heat Transfer, Part B 49 (2006) 259-276.

20. O. Touazi, E. Chénier, R. Eymard, Simulation of natural convection with the collocated clustered finite volume scheme, Comput. Fluids 37 (2008) 11381147.

21. K. Goda, A multistep technique with implicit difference schemes for calculating two- or three-dimensional cavity flows, J. Comput. Phys. 30 (1979) 76 95.

22. H. Sun, R. Li, E. Chénier, G. Lauriat, J. Padet, Optimal plate spacing for mixed convection from an array of vertical isothermal plates, submitted to Int. J. Therm. Sci. (2011).

23. C. Padet, E. M. Mladin, J. Padet, A. Dobrovicescu, Minimization of entropy production in fully developed mixed thermal convection, in: Int. Symp. on Convective Heat and Mass Transfer in Sustainable Energy, 28 April - 1 Mai 2009, Tunisia, CD-ROM paper 69.

\section{List of Tables}

1 Convective heat flux, enthalpy heat flux and mean velocity according to the mesh refinement $\left(n_{z}=20 n_{x}\right)$, for $D / 2=1 \mathrm{~cm}$ and $H=1 \mathrm{~m}$. Order $s$ of the numerical scheme and Richardsonextrapolated values (see Eq. 14). . . . . . . . . . . . .

\section{List of Figures}

1 The physical model of mixed convection in a vertical channel. The full boundary conditions are written in Eqs. (5-7) for the three cases investigated. . . . . . . . . . . . . . . .

2 Variations of the pressure difference between inlet and outlet sections according to the prescribed inlet velocity for forced, mixed and natural convection. . . . . . . . . . . . . . . .

$3 \quad$ Flow recirculation for mixed convection (a) prescribed inlet velocity , $w_{0}=0.05 \mathrm{~m} / \mathrm{s}(\mathrm{b})$ overpressure at the outlet section $p_{s}=1.4 \mathrm{~Pa}$ $(D=3 \mathrm{~cm}, H=1.5 \mathrm{~m}, \Delta T=20 \mathrm{~K}) \ldots \ldots \ldots \ldots$

4 Vertical velocity variations along the channel axis for forced and mixed convection according to the prescribed inlet velocity. The natural convection curve is also shown $(D=2 \mathrm{~cm}, H=1 \mathrm{~m}$, $\Delta T=20 K) \ldots \ldots \ldots \ldots \ldots \ldots \ldots \ldots \ldots \ldots \ldots \ldots \ldots$ 
5 Total heat flux transferred at one of the hot walls for forced and mixed convection according to the prescribed inlet velocity. The value for natural convection is also shown $(D=2 \mathrm{~cm}, H=1 \mathrm{~m}$, $\Delta T=20 K) \ldots \ldots \ldots \ldots \ldots \ldots \ldots \ldots \ldots \ldots \ldots$

6 Axial variation of pressure along the channel axis according to the outlet pressure drop $(D=2 \mathrm{~cm}, H=1 \mathrm{~m}, \Delta T=20 \mathrm{~K})$. The arrows indicate the positions (in $m$ ) of the pressure minimum. . . .

$7 \quad$ Variations of the average velocity according to the pressure drop at the outlet section for forced and mixed convection $(D=2 \mathrm{~cm}$, $H=1 m, \Delta T=20 K)$. . . . . . . . . . . . . . . .

8 Variations of the heat flux transferred at one of the walls according to the pressure drop at the outlet section for forced and mixed convection $(D=2 \mathrm{~cm}, H=1 \mathrm{~m}, \Delta T=20 \mathrm{~K}) . \ldots \ldots$

9 Variations of the temperature along the channel axis according to the pressure drop at the outlet section for natural and mixed convection $(D=2 \mathrm{~cm}, H=1 \mathrm{~m}, \Delta T=20 \mathrm{~K})$. . . . . . . . . .

10 Variations of the vertical velocity component along the channel axis according to the pressure drop at the outlet section for natural and mixed convection $(D=2 \mathrm{~cm}, H=1 \mathrm{~m}, \Delta T=20 \mathrm{~K})$. . . . . . 